\title{
PIXEF: The Livermore PIXE Spectrum Analysis Package
}

\author{
A. J. Antolak \\ G. S. Bench
}

This paper was prepared for submittal to the Atomic Collision in Solids Conference

Ontario, Canada

July 26-30, 1993

July 1293

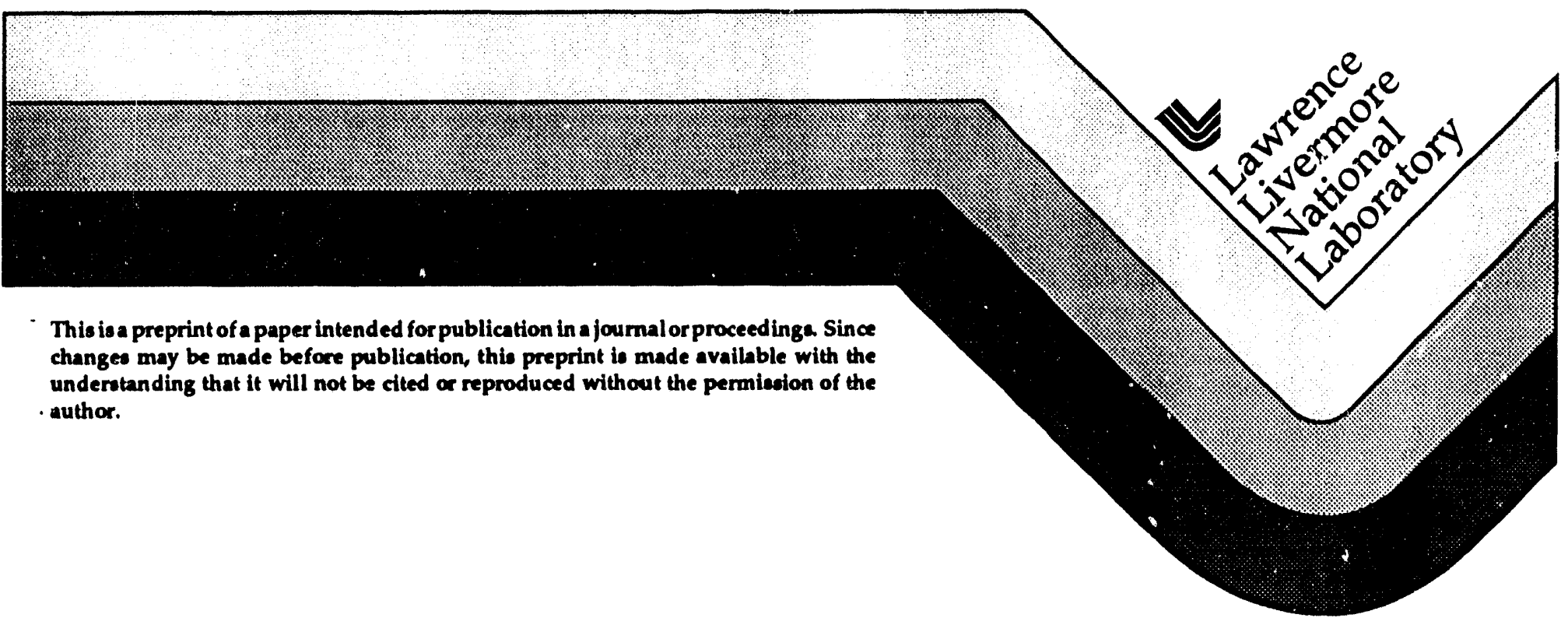




\section{DISCLAIMER}

This document was prepared as an account of work sponsored by an agency of the United States Government. Neither the United States Government nor the University of California nor any of their employees, makes any warranty, express or implied, or assumes any legal liability or responsibility for the accuracy, completeness, or usefulness of any information, apparatus, product, or process disdosed, or represents that its use would not infringe privately owned rights. Reference herein to any specific commercial products, process, or service by trade name, trademark, manufacturer, or otherwise, does not necessarily constitute or imply its endorsement, recommendation, or favoring by the United States Government or the University of California. The views and opinions of authors expressed herein do not mecessarily state or reflect those of the United States Government or the University of California, and shall not be used for advertising or product endorsement purposes. 


\title{
PIXEF : The Livermore PIXE Spectrum Analysis Package
}

\author{
A. J. Antolak ${ }^{\dagger}$ and G. S. Bench $\S$ \\ tSandia National Laboratories, Livermore, CA 94551 \\ §Lawrence Livermore National Laboratory, Livermore, CA 94550
}

\begin{abstract}
PIXEF (for, PIXE-Fit) is a proton-induced $x$-ray emission (PIXE) data analysis program designed for analyzing medium to heavy element matrices while retaining the capability to treat lower atomic number targets. Using nonlinear least squares fitting techniques, algorithms have been developed or modified both for fitting the characteristic $x$-ray peaks and representing the associated bremsstrahlung and $\gamma$-ray background. Self-absorption and secondary fluorescence are explicitly determined for $K$ shell and $L$ subshell $x$ rays. Data bases have been created or improved, where necessary, from reliable current literature values or by direct measurement for element mass attenuation coefficients, photoionization and proton ionization cross sections, Coster-Kronig transition probabilities, fluorescence yields, and relative line intensities. The utility of the program is demonstrated with PIXE spectra obtained at Livermore.
\end{abstract}


reliable approximation to the background. PIXEF uses a combination of peak filing techniques and a statistics-sensitive filtering algorithm to compute the background. Non-linear least squares are used to fit either Gaussian distributions modified by exponential tailing functions or Hypermet ${ }^{3}$ distributions to the measured peak shapes which can then be numerically integrated to provide elemental $x$-ray yields from which quantitative concentration data are obtained. The program is also capable of processing $\mathbf{x}$-ray data from numerous similar spectra by initially specifying a set of experimental input parameters. The following sections present a more detailed description of PIXEF and demonstrate the utility of the software package.

\section{SPECTRUM ANALYSIS}

\section{PIXE Background}

Analogous to other PIXE analysis codes, PIXEF initially computes an approximation to the background continuum. In our experience, we have found that peak filing together with some form of spectrum filtering provides the most reliable and robust method to estimate the background. Although not yet implemented, we have tentative plans to develop a more realistic background model based on physical processes rather than arbitrary fitting functions. One of our intents in developing this software was to make it as self-automated as possible without the need for the user to resort to iterating between various parameters in order to obtain an accurate representation of the spectrum. Currently, PIXEF has options for two different peak filing strategies. One approach follows the SNIP procedure described by Ryan $^{4}$ while the other uses a modified version of Clayton's method. ${ }^{5}$ We have modified the original SNIP algorithm to provide a somewhat better approximation to the types of PIXE spectra we obtain in Livermore, but retained the overall generality of the analysis technique. The modifications include the use of an iteration parameter and a weighting factor in the peak clipping loop. On rare occasions, we have found that the SNIP-like technique can slightly underestinate the magnitude of the background contribution in regions where only weak intensity $x$-ray lines are superimposed on a large or rapidly changing background (typically below $5 \mathrm{keV}$ ). In these cases, the user can optionally implement the alternate Clayton-based method to approximate the background. We have extended Clayton's original technique by applying a statistics-sensitive smoothing filter to the spectrum prior to peak filing and modifying the sampling, weighting and iteration parameters in the peak filing. The smoothing filter helps to improve the fit in regions of the spectrum with poor counting statistics. The Clayton approach has the occasional drawback of noticeably exaggerating the contributions to the background in regions where there are clusters of intense $x$ ray lines and, consequently, the SNIP-based technique is usually preferred. 


\section{Self-Absorption, Secondary Fluorescence and Cross Sections}

Once the calculated background is determined, it is subtracted from the raw spectral data and the resulting $\mathrm{x}$-ray peaks are fitted to either Gaussian or Hypermet ${ }^{3}$ distributions using nonlinear least squares. ${ }^{6}$ At this stage, it is crucial to use accurate relative line intensities in order to obtain a meaningful fit. For this reason, we explicitly calculate target self absorption, secondary fluorescence and ion-induced $x$-ray production cross sections for $K$ and $L$ shell $x$ rays as the ion beam slows down in the specimen matrix. We use the ionization cross sections computed via the ECPSSR model described by Cohe. and Harriganı. ${ }^{7}$ Rather than supplying long tabular data lists in PIXEF with the need to interpolate between values, we utilize a analytic functional fit to the energy-dependent ionization cross sections for each element's $\mathrm{K}$-shell or Lsubshell,

$$
\sigma_{I}(E)=\exp \left(\sum_{n=1}^{8} a_{n} E^{n-1}\right)
$$

where $\sigma_{I}$ is the cross section as a function of energy $E$. We have found the above fits to be typically accurate to within $2 \%$ of the calculated values over the energy range 0.1 to $6.0 \mathrm{MeV}$. Proton stopping powers used to compute the ion slowing down in the specimen matrix are obtained from the expressions given by Andersen and Ziegler. ${ }^{8}$ Self-absorption is treated explicitly for all $\mathrm{K}$ and $\mathrm{L} \mathrm{x}$-ray lines between 1 and $40 \mathrm{keV}$ by incorporating the semi-empirical fits of the mass attenuation coefficients derived by Thinh and Leroux ${ }^{9}$ into PLXEF. Secondary fluorescence can create a significant enhancement to the observed $x$-ray yield. We have followed the mathematical formalism of Reuter to evaluate the secondary fluorescence contributions to each elemental $x$-ray line intensity. ${ }^{10}$ In PIXEF, the subshell and total photoelectric cross sections defined in Reuter's theory are determined directly from the DiracHartree-Slater calculations of Scofield. ${ }^{11}$ Similar to the ionization cross sections above, a fit is made over the $\mathrm{x}$-ray energy range 1 to $150 \mathrm{keV}$ according to

$$
\log \sigma_{P E}(E)=A_{0}+\sum_{n=1}^{2} A_{n} e^{\left(E-E_{0}\right) / B_{n}}
$$

where the left hand side is the logarithm (base 10) of the subshell photoelectric cross section $\sigma_{\mathrm{PE}}$ and, on the right hand side, $E_{0}$ is an adjustable reference energy obtained as an additional fitting parameter along with the $A_{n}$ and $B_{n}$ coefficients. We have found these fits to be typically accurate to within $3 \%$ of the calculated values for $\mathrm{K}$-shell and $\mathrm{L}$-subshell photo ionization. $\mathrm{K}$ shell and $\mathrm{L}$ subshell absorption edges used in the calculation of secondary fluorescence are obtained from McMaster et al.12 The conversion from ionization cross-sections to $\mathrm{x}$-ray production cross-sections requires values for the $\mathrm{x}$-ray fluorescence yields and Coster-Kronig transition probabilities. For the $\mathrm{K}$ (elements $\mathrm{Na}$ to $\mathrm{Ba}$ ) and $\mathrm{L}$ subshell (elements $\mathrm{Ge}$ to $\mathrm{Pu}$ ), we 
use the fluorescence yields and Coster-Kronig values from Chen et al. or Krause. ${ }^{13}$ Relative Kshell and $\mathrm{L}$ subshell $\mathrm{x}$-ray emission rates are obtained either from the compilations of Johansson and Campbell ${ }^{7}$ and the calculations of Cohen and Harrigan ${ }^{14}$ for $3 \mathrm{MeV}$ protons or from our measured values on thin film standards when the relative intensities differ from our own experimental data. Like other groups, we have generally found the greatest discrepancies between measured and calculated relative line intensities for high atomic number $L$ lines. $X$-rays emitted from the sample can also be attenuated by the beryllium window, gold contact layer, and ice layers associated with the $\mathrm{Si}(\mathrm{Li})$ detector while tailing effects on the lineshapes can arise from incomplete charge collection near the edges of the crystal volume. We have measured these absorbing layer thicknesses and the incomplete charge collection effect using calibration standards to physically model our detector with a modification of the formalism given by Cohen. ${ }^{15}$ The modification takes into account the increased attenuation of $x$-rays which enter the detector at non-normal angles of incidence. Over the $x$-ray energy range 1 to $30 \mathrm{keV}$, the $x$-ray detection efficiency curve obtained from this model agrees with experimental PIXE data to within $4 \%$. In a similar manner, we model any additonal $x$-ray filters used to attenuate intense $x$ rays lines using the same approach. PIXEF has the capability of handling up to 10 different types of filters each one with a pinhole if required. Overall, the $x$-ray detection efficiency curves obtained with the use of these model filters agrees with experimental data to within $5 \%$. We have found that the calculated relative line intensities using the above description have provided reliable and accurate results. We are currently considering a more detailed description of the lineshape tailing caused by incomplete charge collection near the front surface of the crystal volume. At present, we do not explicitly calculate matrix effects on M-shell x rays. Secondary fluorescence effects and variation of $\mathbf{M}$-shell proton ionization cross sections with ion energy are neglected and $\mathrm{M}$-shell relative emission rates are only approximately corrected for $\mathrm{x}$-ray absorption in the matrix. As a result, we do not compute $\mathrm{M}$-shell $\mathrm{x}$-ray yields for quantitative analysis but include these lines in the fit of the spectra for completeness.

\section{X-ray Line Shapes, Fitting Procedure and Yield Calculation.}

The response function of the $\mathrm{Si}(\mathrm{Li})$ detection system yields a Gaussian-like distribution for the emitted Lorentzian-shaped $x$-ray lines. For well-resolved lines, the convolution of these distributions (i.e., a Voigt function) is the best representation for the characteristic peak shape. In PIXEF, two options are presently available to represent the basic $x$-ray line shapes. The first option uses a Gaussian modified by a low-energy exponential tail. The second option utilizes the Hypermet $^{3}$ description which includes exponential tailing convoluted with a Gaussian for the detector response leading to the product of an exponential and a complementary error function.

With both options a step function is implemented which accounts for the disproportionate 
response in the tailing between the $K_{\alpha}$ and $K_{\beta}$ lines. We have generally found the Hypermet representation to yield slightly smaller $\chi^{2}$ residuals in the fit compared to those obtained from the more simple exponential form for tailing especially for targets containing higher atomic number elements. However, the increased accuracy also comes at the expense of a slighly longer execution time in the least squares fitting algorithm due to the required numerical evaluations of the complementary error functions. We are currently investigating the use of Voigtian functions to represent peak line shapes in multi-element spectra and would expect further improvement in the overall fits. Since many of the $\mathrm{x}$-ray lines in our data base occur in pairs such as $\mathrm{K}_{\boldsymbol{\alpha}_{1}}$ and $K_{\alpha_{2}}$, the $x$-ray centroid energy is given by $\mu=A+B E_{k}$ with width $\sigma=\left(C^{2}+D^{2} E_{k}\right)^{1 / 2}$ where $E_{k}$ is the energy of the $k$-th $x$-ray line of the element. The $K_{\alpha}$ peak is fitted in PIXEF with both $K_{\alpha_{1}}$ and $K_{\alpha_{2}}$ relative line intensities; likewise, the $L_{\alpha}$ peak is treated in the same manner. The user has the option to specify on which elements peak tailing occurs.

$\mathrm{X}$-rays generated by the target with energies greater than the silicon absorption edge can fluoresce silicon atoms in the $\mathrm{Si}(\mathrm{Li})$ detector and silicon $\mathrm{x}$ rays escape from the active volume. Although these counts are lost by the parent $x$-ray peaks, they reappear in the spectrum as new peaks with energies $E_{\text {parent }}-1.74 \mathrm{keV}$. Cohen and Clayton have shown that the logarithm of escape to parent peak can be accurately fitted to a quadratic in $\mathrm{E}_{\text {parent. }}{ }^{16}$ We have measured our own escape to parent peak ratio over the energy range 2 to $15 \mathrm{keV}$ and have also fitted the data to a quadratic. In PIXEF, the silicon escape peaks are stripped from the raw spectrum prior to the background fit and subseqiently added back to the computed spectrum after the least squares fitting is complete. Additionally, at high count rates, two x-rays entering the detector at about the same time can be recorded as one pulse. In the spectrum analysis, these pileup peaks are treated symbolically in PIXEF as elements with the position and relative intensities of their characteristic $x$-ray lines computed as described by Johansson. ${ }^{17}$ The pileup energies are included in the fitting algorithm and identified in the final output. The user can select the number of pileup peaks to include in the analysis and for what elements they are to be associated.

The standard nonlinear least squares method as formulated in Bevington's program ${ }^{6}$ is used to fit the model peak shapes to the experimental data with the goodness-of-fit measured by chi-squared $\left(\chi^{2}\right)$. Assuming uncertainties arise only from counting statistics, the weighting factors in the $\chi^{2}$ expression are simply the inverse of the experimental data. However, following the recommendation of Phillips and Marlowe ${ }^{3}$ in regions of low counting statistics (i.e., when the number of counts in channel $c_{i}$ is $<10$ in PIXEF), the weight is computed either as the inverse of the three channel average $\left(c_{i-1}+c_{i}+c_{i+1}\right) / 3$ if the average is greater than one or set to unity.

The output from PIXEF is the $K_{\alpha_{1,2}}$ or $L_{\alpha_{1,2}}$ yield that has been corrected for secondary fluorescence. The above data bases are used to calculate the elemental concentrations from the standard integral expression 


$$
y=\frac{1}{4 \pi} \frac{C N_{A} \Omega q \varepsilon}{A e} \int_{E_{0}}^{0} \frac{\sigma(E) d E}{S(E)} \exp \left(-\mu \frac{\cos \theta_{b}}{\cos \theta_{d}} \int_{E_{0}}^{E} \frac{d \tilde{E}}{S(\tilde{E})}\right)
$$

where $y$ is the $x$-ray yield for an element of concentration $C, N_{A}$ is Avagadro's number, $\Omega$ is the solid angle subtended by the detector, $q$ is the collected charge, $\varepsilon$ is the detector efficiency, $A$ is the element's atomic weight, $e$ is the charge of an electron, $E_{0}$ is the incident proton beam energy, $\sigma(E)$ is the $x$-ray production cross section computed from the fluorescence yield, Coster-Kronig transition probabilities and ECPSSR ionization cross section, $S(E)$ is the proton stopping power in the specimen matrix, and $\theta_{b}$ and $\theta_{d}$ are the angles between the sample normal and, respectively, the incoming beam and detector. We have tested PIXEF on spectra collected with our system from several standards and have found it to produce agreement with the certified values to within $4 \%$ in general.

\section{ILLUSTRATIVE EXAMPLES}

In this section, we present some examples of the kinds of PIXE data we routinely analyze in Livermore. The examples were selected in order to demonstrate the typical output produced by the PIXEF software as well as giving an indication of the accuracy one can expect from the fitting procedure. In all cases, the targets were bombarded with a 3-MeV proton beam focussed to a spot size between 10 and $200 \mu \mathrm{m}$ and the data collected with a maximum beam current of up to $1 \mathrm{nA}$. The raw PIXE data are shown in the figures as a dotted line, the fit to the background by a dashed line and the fit to the peaks by a solid line.

Figure 1 shows the measured and calculated PIXE spectra from a thin sample containing uranium. We have found that the published relative intensities for uranium L-lines led to inaccuracies in both the final spectrum fit and the computed elemental concentration levels. The fit to the peaks shown in the figure (solid line) was obtained by subsequently incorporating the relative intensities of 18 uranium L-lines measured in our laboratory. Not using all L-lines in the analysis may partially account for the observed small discrepancies. From the analysis, we measure the $U$ thiskness to be $33.2 \pm 3.0 \mu \mathrm{g} / \mathrm{cm}^{2}$ compared to a nominal supplied value of $32.8 \pm$ $1.7 \mu \mathrm{g} / \mathrm{cm}^{2}$. The unfitted portion of the spectrum near $11 \mathrm{keV}$ corresponds to $\mathrm{Ta} L \gamma$-lines in the target. The doublet at around $22 \mathrm{keV}$ is poorly fit because the present PIXEF data base improperly represents it as a single $x$-ray line.

The PIXE spectra from a Livermore environmental air filter sample are shown in Figure 2. These samples are collected and analyzed to determine lead concentrations in the atmosphere near the laboratory. This example is indicative of PIXEF's capability to accurately fit a large number of elements over a broad dynamic range. Our analysis gives a total $\mathrm{Pb}$ concentration on 
the filter of $0.16 \pm 0.03 \mu \mathrm{g} / \mathrm{cm}^{2}$ which agrees to within $10 \%$ of values obtained by other analytical techniques.

In Figure 3 are the PIXE spectra obtained from a thick zinc - nickel calibration sample. We used this sample to calibrate our measurements of thin Ni- $\mathrm{n}$ electroplated films on Hull cell test strips. The latter are used by the electroplating industry to measure physical properties and variations in the plated films as a function of the applied current across the strip. Materials Research Corporation (MRC) of Orangeburg, N.Y. who supplied the standard sample specified the nickel content as $29.9 \%$ by weight. Individual element lines are identified in the spectra. Explicitly including target self-absorption and secondary fluorescence effects yields a nickel concentration of $30.9 \pm 1.7 \mathrm{wt} \%$. If secondary fluorescence effects are neglected, the calculated nickel concentration would be $38.1 \pm 2.3 \mathrm{wt} \%$. Likewise, the zinc, iron and chromium concentrations agree well with the MRC specifications when fluorescence contributions are taken into account.

Figure 4 shows PIXE spectra obtained from a spot in a linescan across a thick section of a mollusk shell with matrix composition approximately $\mathrm{CaCO}_{3}$. The sample was examined to determine if the $\mathrm{Sr}$ to $\mathrm{Ca}$ ratio can be correlated to water temperature or salinity. The data were collected with a $0.76 \mathrm{~mm}$ mylar filter to attenuate the intense $\mathrm{Ca} \times$ rays. The $\mathrm{Ca}$ concentration determined from these spectra was $38 \pm 3$ wt\% consistent with the $\mathrm{CaCO}_{3}$ matrix composition and the $\mathrm{Sr}$ concentration agreed with the results of other analysis techniques. The background fit was determined using the Clayton-based background algorithm since the SNIP-like approach underestimated the background in the region between 1 and $8 \mathrm{keV}$. In the latter case, the reduced background caused $\mathrm{x}$-ray yields from $\mathrm{Mn}, \mathrm{Fe}$ and $\mathrm{Cu}$ to be significantly overestimated. The concentrations obtained from the fit displayed in figure 4 are in good agreement with those obtained by other analytical techniques.

Figure 5 shows PIXE spectra from a $\mathrm{HgI}_{2}$ crystal sample designed for use as a room temperature $\gamma$-ray detector. The sample was studied in order to identify and quantify any trace elements which may affect detector performance. To attenuate the intense iodine L-lines a 0.76 $\mathrm{mm}$ mylar filter was placed in front of the detector. The $\mathrm{Hg}$ and I concentrations determined from these data agree well with the sample stoichiometry. The measured $\mathrm{Fe}$ and $\mathrm{Ni}$ concentrations seen in the figure are approximately an order of magnitude higher than those determined by inductively coupled plasma atomic emission spectrometry (a destructive bulk analysis technique) indicating that there may be surface contamination of this sample. In PIXE analyses on similar calibration samples, the concentrations of the $\mathrm{Fe}$ and $\mathrm{Ni}$ trace impurities were comparable to the levels obtained by other methods so we do not believe that there is some type of systematic error in PIXEF for $\mathrm{HgI}_{2}$-type samples. 


\section{CONCLUSION}

We have described the sofiware package PIXEF presently used and supported in Livermore to analyze particle-induced x-ray emission data simply, reliably and accurately. PIXEF is part of the comprehensive Ion Micro-Analysis Package (IMAP) for analysis of nuclear microprobe data acquired from PIXE, ion backscattering spectroscopy (IBS), particle-induced gamma emission (PIGE), scanning transmission ion microscopy (STIM) and ion microtomography (IMT). 18 Although PIXEF can handle a limited form of layered target geometry (up to 6 layers), we plan to extend PIXEF to properly treat layered media for both $\mathrm{K}$ and $\mathrm{L}$ lines. Further refinements are needed to accurately fit peaks with very poor counting statistics. As alluded to early in the paper, we plan to improve the spectral description by implementing a more realistic physical model for the background and develop a more realistic representation of the $x$-ray lineshapes by incorporating Voigtian distributions in the peak fitting routines and, because of their importance for heavier elements, explicitly calculate matrix effects for M-shell x rays.

\section{ACKNOWLEDGEMENTS}

This work supported by the U. S. Department of Energy under SNL Contract No. DE-AC0476P00789 and LLNL Contract No. W-7405-ENG-48. The authors would also like to thank A. Friz for generating some of the data bases. 


\section{REFERENCES}

${ }^{1} U$. Wätjen, Nucl. Instr. Meth. B22 (1987) 29.

2J. L. Campbell, W. Maenhaut, E. Bombelka, E. Clayton, K. Malmqvist, J. A. Maxwell, J. Pallon and J. Vandenhaute, Nucl. Instr. Meth. B14 (1986) 204.

${ }^{3}$ G. W. Phillips and K. W. Marlow, IEEE Trans. Nucl. Sci. NS-24 (1977) 154.

${ }^{4}$ C. G. Ryan, E. Clayton, W. L. Griffin, S. H. Sie and D. R. Cousens, Nucl. Instr. Meth. B34 (1988) 396.

SE. Clayton, PIXAN - The Lucas Heights PIXE Analysis Package, Australian Atomic Energy Commission, Report AAEC/M113 (1986).

6P. R. Bevington, Data Reduction and Error Analysis for the Physical Sciences, McGraw-Hill, New York, 1969.

7S. A. E. Johansson and J. L. Campbell, PIXE: A Novel Technique for Elemental Analysis, John Wiley \& Sons, Chichester, 1988; D. D. Cohen, Australian Nuclear Science \& Technology Organisation, private communication; D. D. Cohen and M. Harrigan, At. Data Nucl. Data Tables 33 (1985) 255.

${ }^{8} \mathrm{H}$. H. Andersen and J. F. Ziegler, Hydrogen - Stopping Powers and Ranges in All Elements, Pergamon Press, New York, 1977.

9J. Leroux and T. P. Thinh, Revised Tables of X-Ray Mass Attenuation Coefficients, Corp. Scient. Claisse Inc., Québec, 1977.

10W. Reuter, A. Lurio, F. Cardone and J. F. Ziegler, J. Appl. Phys. 46 (1975) 3194.

11J. H. Scofield, Lawrence Livermore National Laboratory Report UCRL-51326, 1976.

12W. H. McMaster, N. Kerr Del Grande, J. H. Mallert and J. H. Hubbell, Lawrence Radiation Laboratory Report UCRL-50174, 1969.

${ }^{13}$ M. H. Chen, B. Crasemann and H. Mark, Phys. Rev. A24 (1981) 177; M. O. Krause, J. Phys. Chem. Ref. Data 8 (1979) 307.

14D. D. Cohen and M. Harrigan, At. Data Nucl. Data Tables 34 (1986) 393.

${ }^{15}$ D. D. Cohen, Nucl. Instr. Meth. 193 (1980) 15.

16D. D. Cohen and E. Clayton, in Ion Beams for Materials Analysis, edited by J. R. Bird and J. S. Williams, Academic Press, Sydney, Australia, 1989.

${ }^{17}$ G. I. Johansson, X.Ray Spectrom. 11 (1982) 194.

${ }^{18}$ A. J. Antolak, G. S. Bench, M. L. Hildner and D. H. Morse, to be published in Nucl. Instr. Meth. 


\section{FIGURE CAPTIONS}

Figure 1. PIXE spectra of a thin sample containing uranium. The raw data is depicted as a dotted line, the calculated background is shown as a dashed line anc he fit to the peaks is the solid line. The unfitted portion of the spectrum near $11 \mathrm{keV}$ corresponds to $\mathrm{Ta} L \gamma$-lines in the target.

Figure 2. The PIXE spectra from a Livermore environmental air filter sample. These samples are collected and analyzed to determine lead concentrations in the atmosphere near the laboratory. The dotted, dashed and solid lines correspond to the same curves used in figure 1.

Figure 3. PIXE spectra obtained from a thick zinc - nickel calibration sample used to calibrate our measurements of thin Ni- $\mathrm{Zn}$ electroplated films on Hull cell test strips. The dotted, dashed and solid lines correspond to the same curves used in figure 1.

Figure 4. The PIXE spectra obtained from a spot in a linescan across a thick section of a mollusk shell with matrix composition $\mathrm{CaCO}_{3}$. The sample was examined to determine if the $\mathrm{Sr}$ to $\mathrm{Ca}$ ratio can be correlated to water temperature or salinity. The dotted, dashed and solid lines correspond to the same curves used in figure 1.

Figure 5. The measured and computed PIXE spectra from a $\mathrm{HgI}_{2}$ crystal sample fabricated as a room temperature $x$-ray detector. The sample was studied in order to identify and quantify any trace elements which may affect detector performance.The dotted, dashed and solid lines correspond to the same curves used in figure 1. 


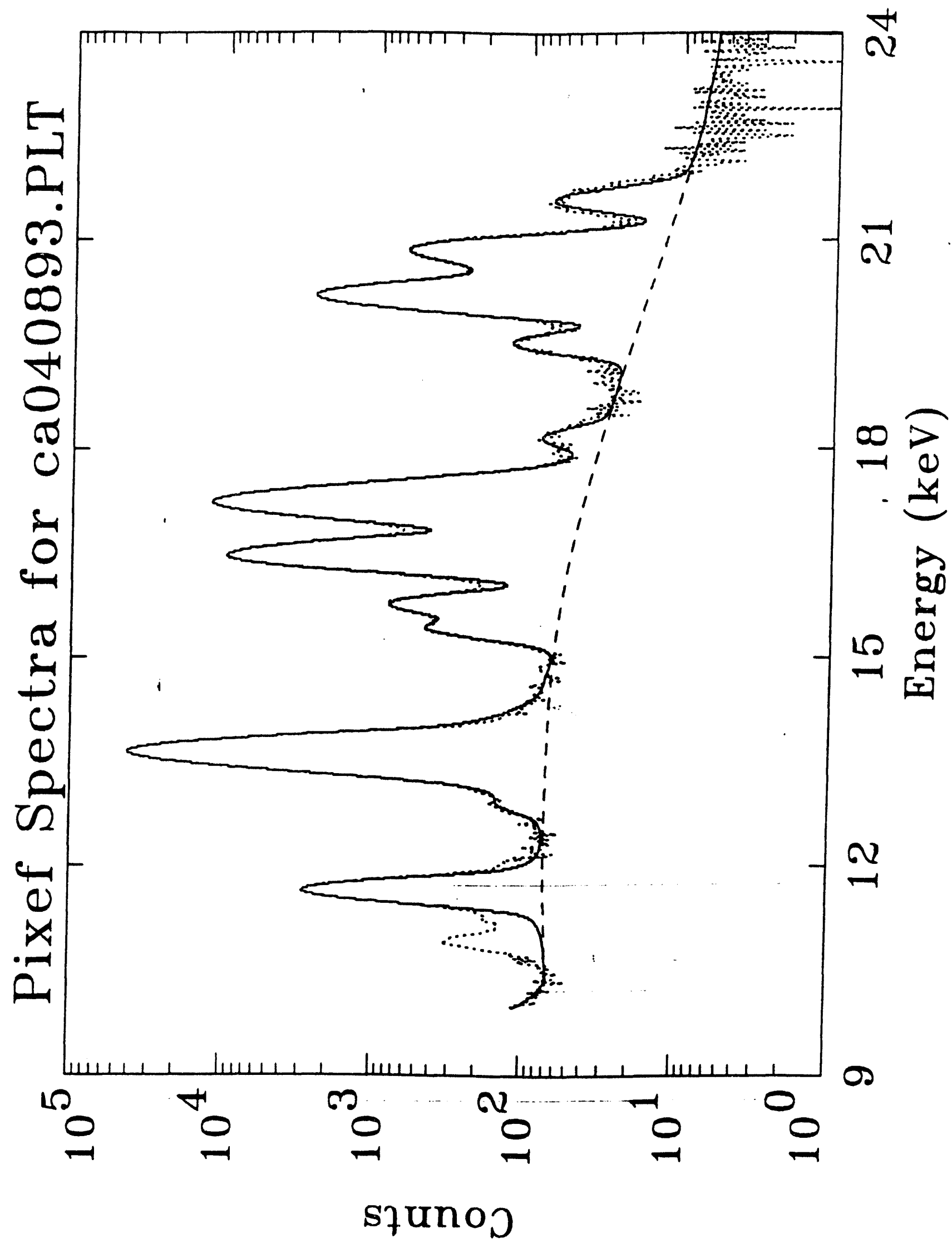

Figl 


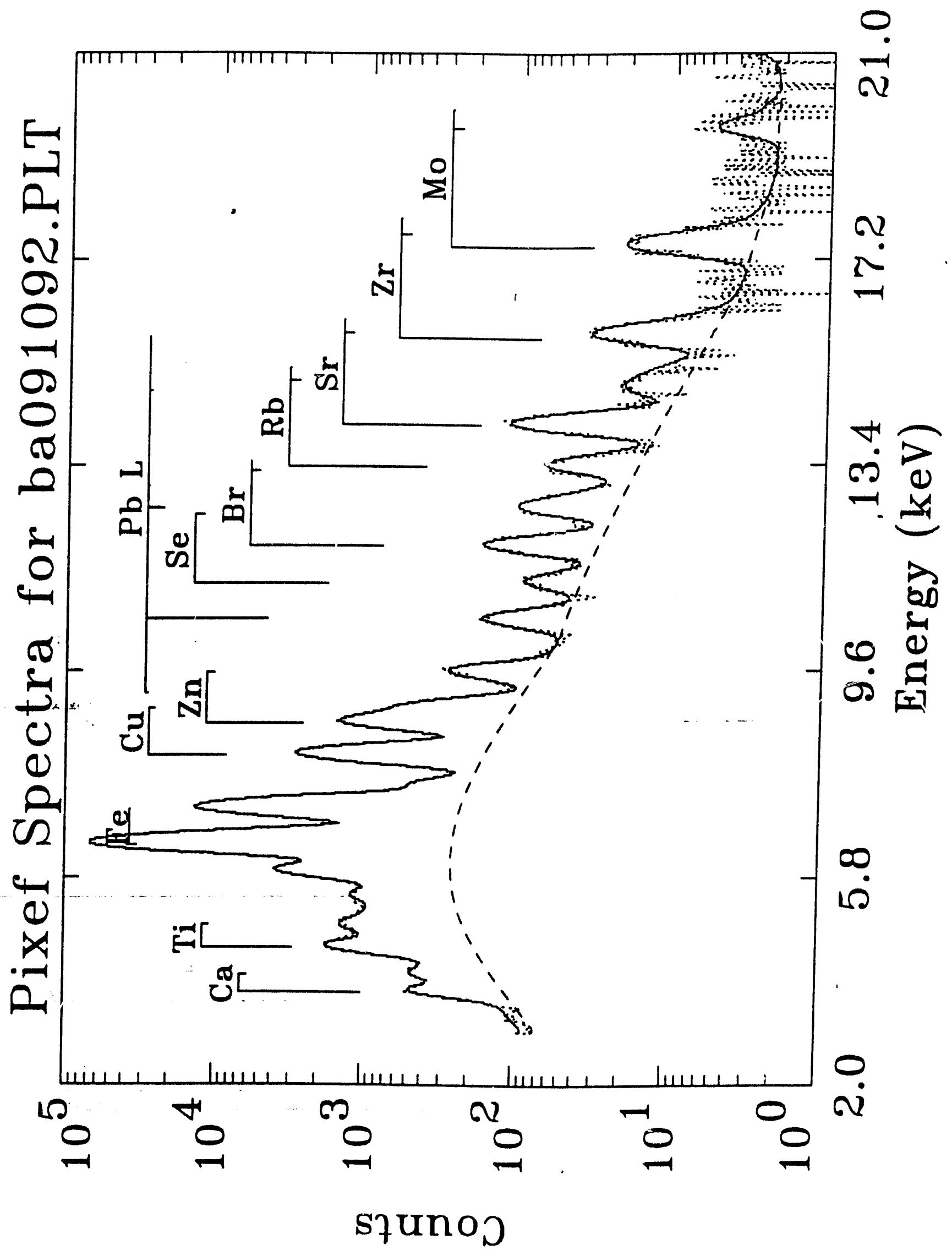

Figh 


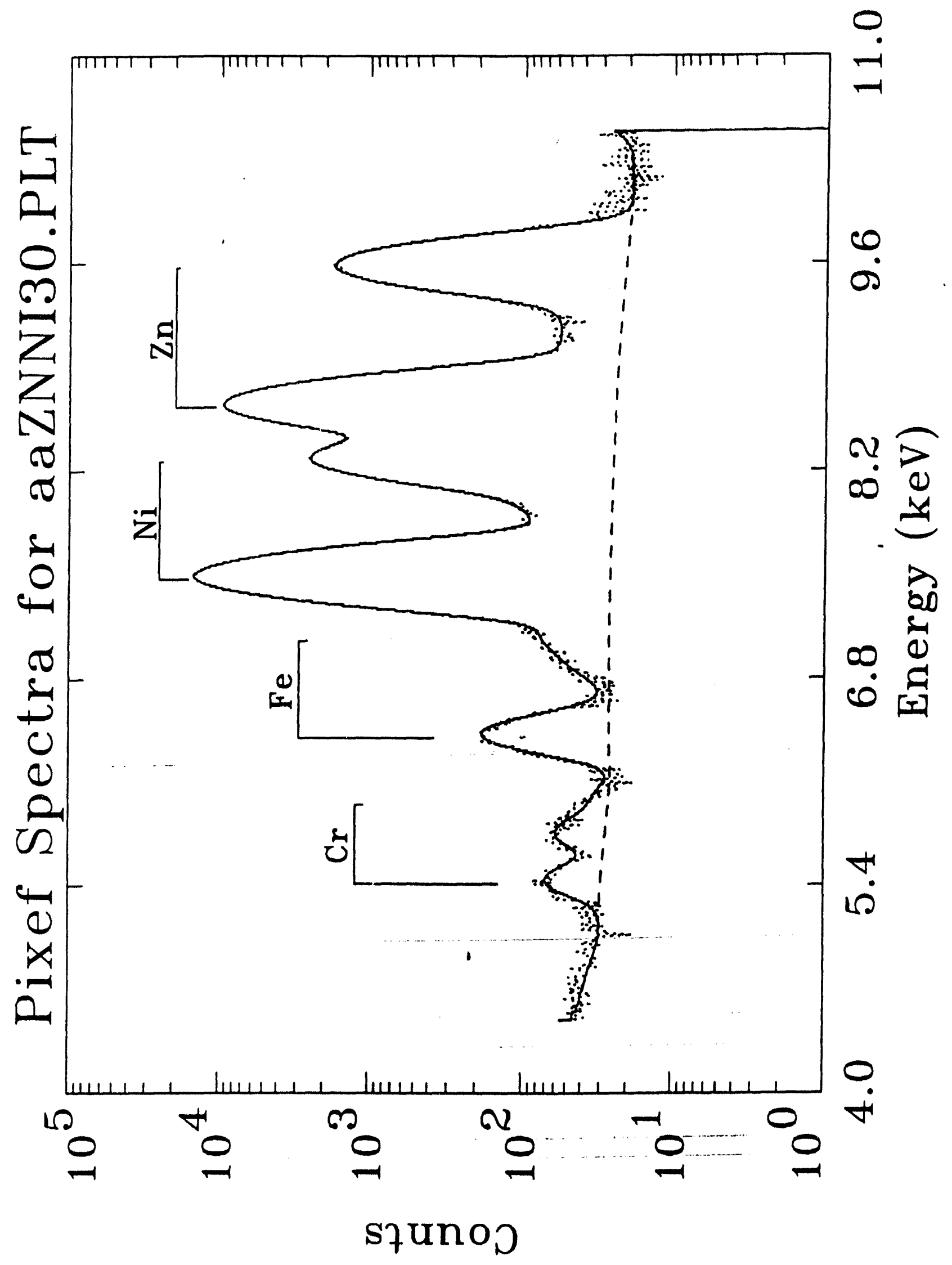

Fig 3 


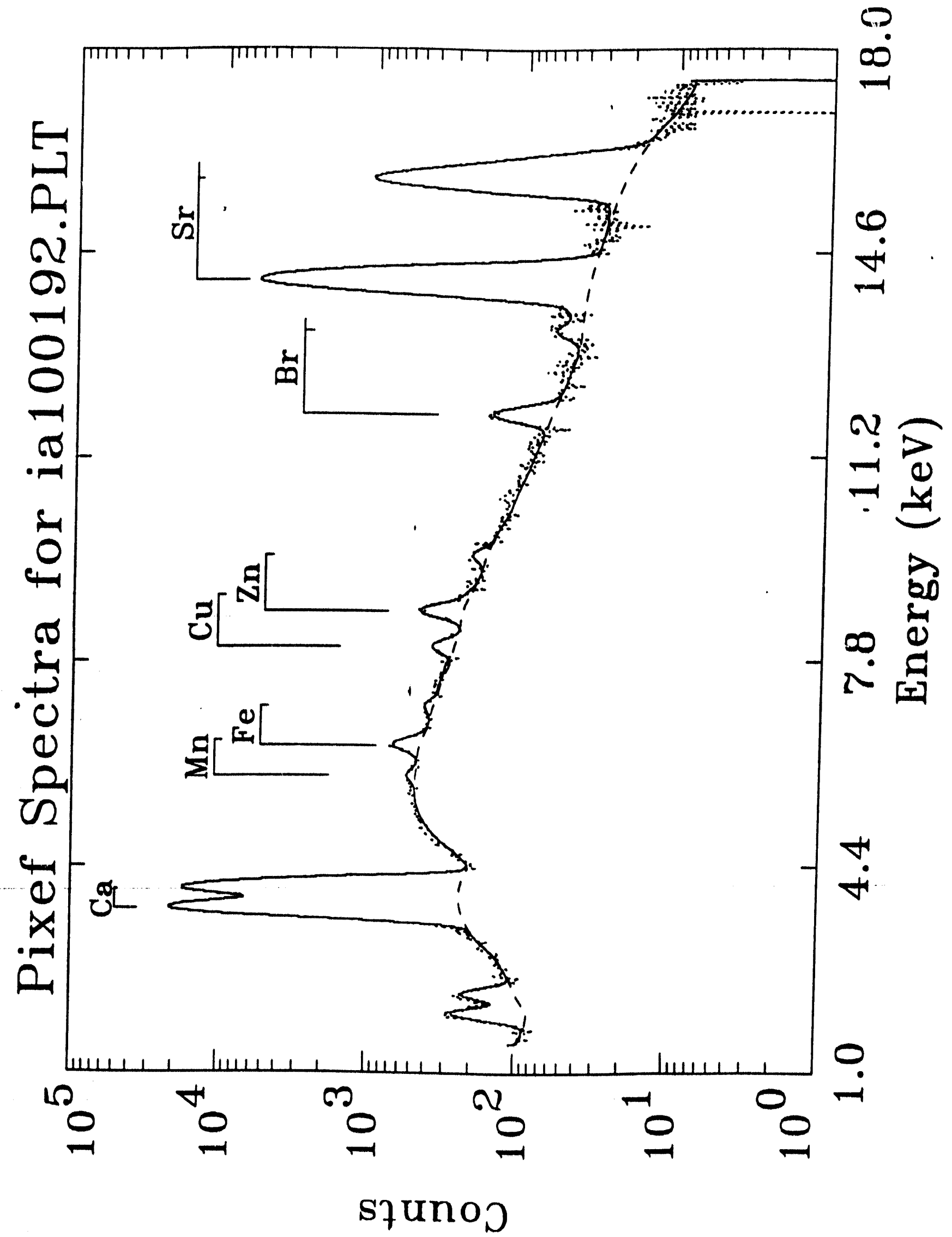

Figl 4. 


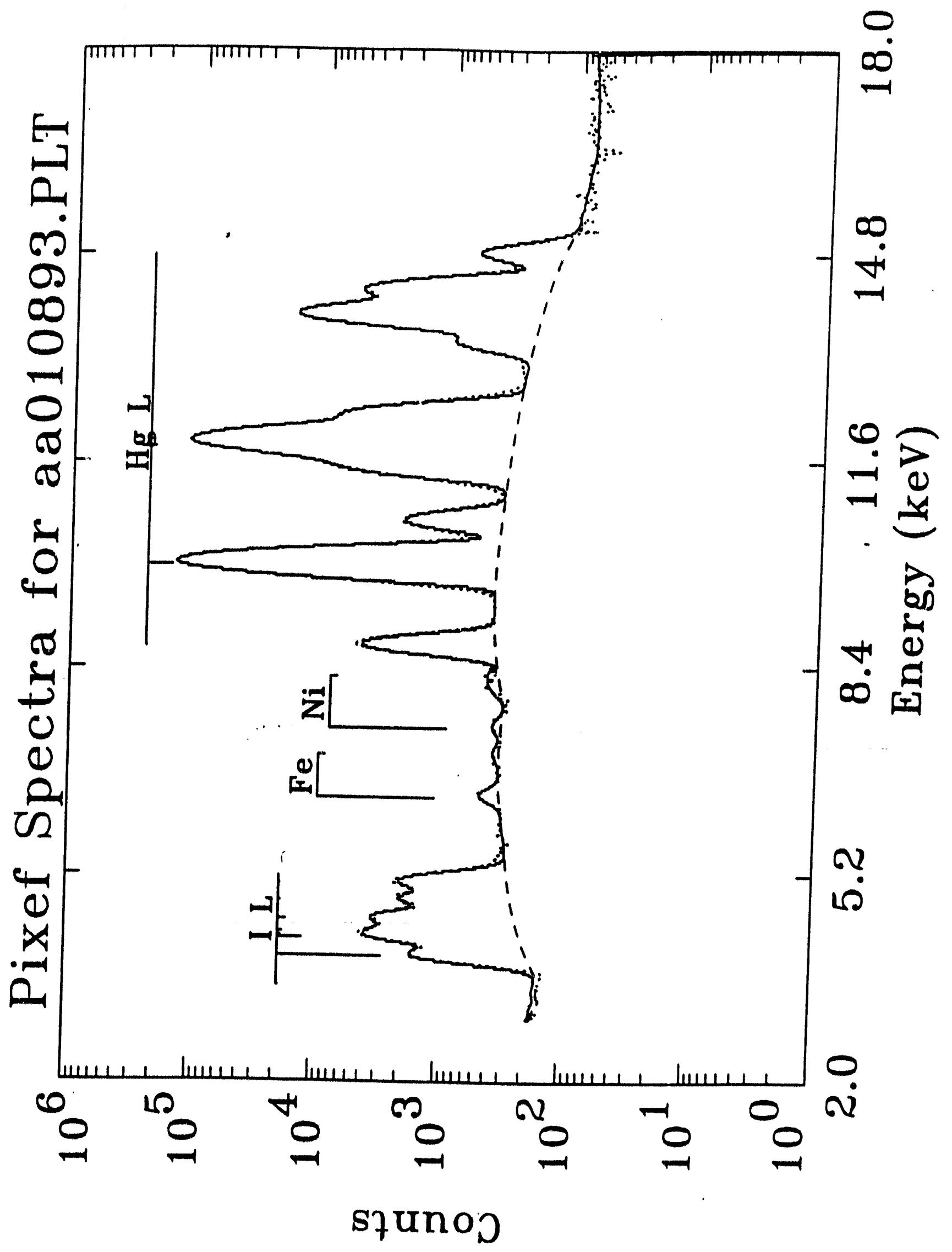

Figs 

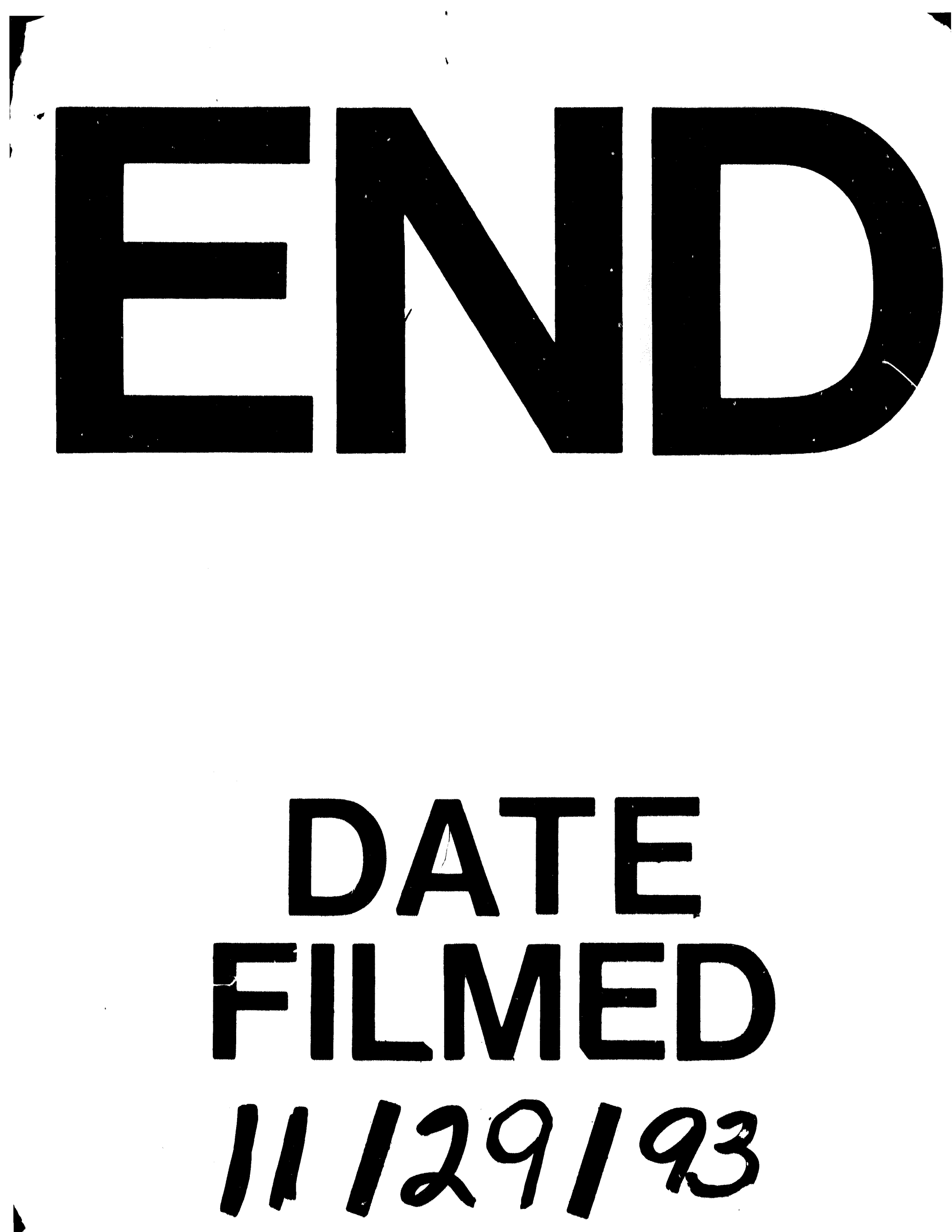
